\title{
Calibration of Electrical Impedance Tomography
}

\author{
W. Daily, A. Ramirez
}

May 1, 2000

U.S. Department of Energy

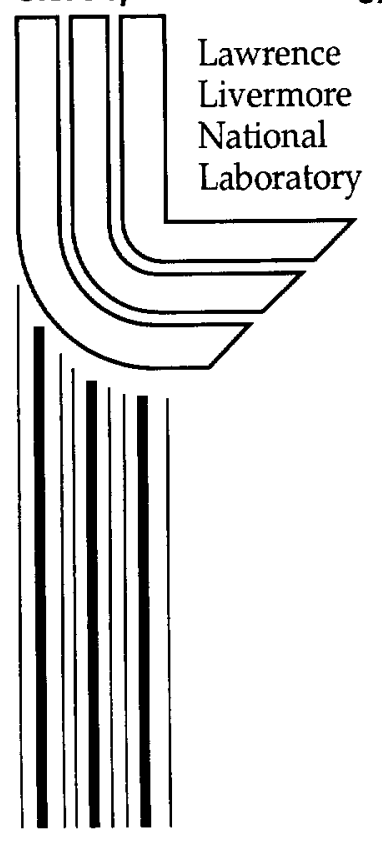




\section{DISCLAIMER}

This document was prepared as an account of work sponsored by an agency of the United States Government. Neither the United States Government nor the University of California nor any of their employees, makes any warranty, express or implied, or assumes any legal liability or responsibility for the accuracy, completeness, or usefulness of any information, apparatus, product, or process disclosed, or represents that its use would not infringe privately owned rights. Reference herein to any specific commercial product, process, or service by trade name, trademark, manufacturer, or otherwise, does not necessarily constitute or imply its endorsement, recommendation, or favoring by the United States Government or the University of California. The views and opinions of authors expressed herein do not necessarily state or reflect those of the United States Government or the University of California, and shall not be used for advertising or product endorsement purposes.

This work was performed under the auspices of the U. S. Department of Energy by the University of California, Lawrence Livermore National Laboratory under Contract No. W-7405-Eng-48.

This report has been reproduced directly from the best available copy.

Available electronically at http://www.doc.gov/bridge

Available for a processing fee to U.S. Department of Energy

And its contractors in paper from

U.S. Department of Energy

Office of Scientific and Technical Information

P.O. Box 62

Oak Ridge, TN 37831-0062

Telephone: (865) 576-8401

Facsimile: (865) 576-5728

E-mail: reports@adonis.osti.gov

Available for the sale to the public from

U.S. Department of Commerce

National Technical Information Service

5285 Port Royal Road

Springfield, VA 22161

Telephone: (800) 553-6847

Facsimile: (703) 605-6900

E-mail: orders@ntis.fedworld.gov

Online ordering: http://www.ntis.gov/ordering.htm

OR

Lawrence Livermore National Laboratory

Technical Information Department's Digital Library

http://www.llnl.gov/tid/Library.html 


\title{
Calibration of Electrical Impedance Tomography
}

\author{
by \\ William Daily and Abe Ramirez
}

May 2000

\section{Introduction}

Over the past 10 years we have developed methods for imaging the electrical resistivity of soil and rock formations. These technologies have been called electrical resistance tomography of ERT (e.g. Daily and Owen, 1991). Recently we have been striving to extend this capability to include images of electrical impedance - with a new nomenclature of electrical impedance tomgraphy or EIT (Ramirez et al., 1999). Electrical impedance is simply a generalization of resistance. Whereas resistance is the zero frequency ratio of voltage and current, impedance includes both the magnitude and phase relationship between voltage and current at frequency. This phase and its frequency behavior is closely related to what in geophysics is called induced polarization or (Sumner, 1976).

Why is this phase or IP important? IP is known to be related to many physical phenomena of importance so that image of IP will be maps of such things as mineralization and cation exchange IP (Marshall and Madden, 1959). Also, it is likely that IP, used in conjunction with resistivity, will yield information about the subsurface that can not be obtained by either piece of information separately.

In order to define the accuracy of our technologies to image impedance we have constructed a physical model of known impedance that can be used as a calibration standard. It consists of 616 resistors, along with some capacitors to provide the reactive response, arranged in a three dimensional structure as in Figure 1. Figure 2 shows the construction of the network and defines the coordinate system used to describe it. This network of components is a bounded and discrete version of the unbounded and continuous medium with which we normally work (the subsurface). The network has several desirable qualities: 1 . The impedance values are known (to the accuracy of the component values). 2 . The component values and their 3D distribution is easily controlled. 3. Error associated with electrode noise is eliminated. 4. Each box formed by 12 adjacent components corresponds to a voxel in the finite difference forward model used in the inverse code and this correspondence makes for easy comparison of inversion results and model physical parameters.

Using this network we can study the errors associated with the measurement system (called Zombie) separated from the errors introduced by electrode noise. We can also learn details in the behavior of the inversion software (called $C R 3 D$ ) by comparing images and model.

\section{Experimental Procedure}

EIT data (magnitude and phase) were acquired on the network in two states: 
1-All components are $1000 \pm 5 \% \mathrm{ohm}$ resistors. This is an (approximately) uniformly resistive target of $1000 \mathrm{ohm} \mathrm{m}$ (when the scale unit is $1 \mathrm{~m}$ ) and of uniform phase $0 \mathrm{mr}$ (there are no reactive components such as capacitors in the network).

2-All components in the network are as in case 1 except for the following two anomalies: the box whose comers are $2,2,2 ; 3,2,2 ; 2,3,2 ; 3,3,2 ; 2,2,3 ; 3,2,3 ; 2,3,3 ; 3,3,3$ has each edge formed by a 1 micro farad capacitor and a $1000 \mathrm{ohm}$ resistor in parallel and the box whose comers are $5,5,3 ; 6,5,3 ; 5,6,3 ; 6,6,3 ; 5,5,4 ; 6,5,4 ; 5,6,4 ; 6,6,4$ has each edge formed by two $1000 \mathrm{ohm}$ resistors in parallel. This arrangement yields a uniformly resistive network of $1000 \mathrm{ohm} m$ except for a single voxel which has a capacitive reactance with a time constant of $\tau=R C=10^{-3} \mathrm{~s}$ and another voxel which has a resistance of $500 \mathrm{ohm} \mathrm{m}$ and no reactance.

A useful description of these anomalies is in the complex plane. Using this convention the impedance is $Z=Z_{r}+j Z_{1}$, where $j=(-1)^{1 / 2}$ and $|Z|=\left(Z_{r}{ }^{2}+Z_{i}{ }^{2}\right)^{1 / 2}$ and $\tan \theta=Z_{i} / Z_{r}$. The resistive anomaly then has $|Z|=R^{2} /(2 R)$ which becomes $500 \mathrm{ohm}$ and $\theta=0$. The reactive anomlay has

$$
|Z|=R /\left(1+\omega^{2} C^{2} R^{2}\right)^{1 / 2}
$$

which at $4 \mathrm{~Hz}$ is $999.7 \mathrm{ohm}$ and

$$
\theta=-\tan ^{-1}(\omega C R)
$$

which at $4 \mathrm{~Hz}$ is $-30 \mathrm{mr}$. Because this is a convenient format to describe the reactance we will display EIT reconstructions of $\mid \mathrm{ZI}$ and $\theta$.

Transfer resistances were measured as though the network where a volume being sampled by electrodes in a series of boreholes along the edges. These boreholes are defined by the series of nodes (each node as though it were an electrode) and are always referenced in pairs as follows:

When referenced first in the series of two holes borehole 1 is nodes $1,1,1 ; 1,2,1 ; 1,3,1 ; 1,4,1$; $1,5,1 ; 1,6,1 ; 1,7,1$. For example, when we refer to hole pair 1,3 then the above nodes define borehole 1 .

When referenced second in the series of two holes borehole 1 is nodes $7,1,1 ; 7,2,1 ; 7,3,1$; $7,4,1 ; 7,5,1 ; 7,6,1 ; 7,7,1$. For example, when we refer to hole pair 3,1 then the above nodes define borehole 1 .

When referenced first in the series of two holes borehole 3 is nodes $1,1,3 ; 1,2,3 ; 1,3,3 ; 1,4,3$; $1,5,3 ; 1,6,3 ; 1,7,3$. For example, when we refer to hole pair 3,1 then the above nodes define borehole 3 .

When referenced second in the series of two holes borehole 3 is nodes $7,1,3 ; 7,2,3 ; 7,3,3$; $7,4,3 ; 7,5,3 ; 7,6,3 ; 7,7,3$. For example, when we refer to hole pair 1,3 then the above nodes define borehole 3 . The other borehole arrays are similarly defined.

Data were measured using a four-point resistance method collecting all the linearly independent data from a dipole-dipole configuration. For each measurement, the reciprocal measurement was also taken. Data from the following borehole combinations were used for each inversion: 1,$1 ; 1,2 ; 1,3 ; 1,4 ; 1,5 ; 2,1 ; 2,2 ; 2,3 ; 2,4 ; 2,5 ; 3,1 ; 3,2 ; 3,3 ; 3,4 ; 3,5 ; 4,1 ; 4,2$; 
4,$3 ; 4,4 ; 4,5 ; 5,1 ; 5,2 ; 5,3 ; 5,4 ; 5,5$. This results in 1925 measured transfer resistances that can be used in the inversion (there are also 1925 reciprocal measurements).

\section{Results and Discussion}

We will examine the results two different ways. First, we will look at the transfer measurements before any inverse processing and this will yield information about the accuracy of the measurement system. Second, we will look at the reconstructed images of impedance and this will reflect the accuracy of the inversion algorithm, as embodied in the computer code CR3D, as well as the accuracy of the data.

\section{Transfer Resistance Accuracy}

A simple test for measurement accuracy is a comparison of the measurement and its reciprocal. This is the standard test we use for accuracy of field data and so we use it here also. The percent difference between a transfer resistance $R_{t}$ and its reciprocal $R_{r}$ or $100\left(R_{t}\right.$ $\left.-R_{\mathrm{T}}\right) / R_{\mathrm{t}}$ is used to compare the two magnitudes and the difference $\theta_{\mathrm{t}}-\theta_{\mathrm{r}}$ is used to compare the phases. Figure 3 shows the reciprocity for a subset of network data at $4 \mathrm{~Hz}$. For comparison Figure 4 shows the reciprocity of $4 \mathrm{~Hz}$ data taken on a target immersed in a tank of water. The experimental conditions are similar to those for the measurements on the network (both $4 \mathrm{~Hz}$ ) except for the absence of electrodes in the measurement circuit for the network data. The network data errors are clearly much smaller than the water tank errors - the magnitude percent differences are about a third of those in the water tank and the phase differences are about half those of the water tank. These comparisons are expected to change somewhat with the experimental details but we believe that these results show that significant measurement error is introduced by the electrodes. We also believe that the network data phase errors at $4 \mathrm{~Hz}$ are less than approximately $1 \mathrm{mr}$.

Another important test of the measurement validity is shown in Figure 5 where we have plotted the measured phase (for all measurements in plane 3,3) verses frequency for the blank (no reactive anomaly) network and for the nonuniform (with capacitor anomaly) network. For the blank case all the components of the network are purely resistive so that the phase should vanish at all frequencies. Nonzero phases reflect errors in measurement, either in the measurement system itself or as a result of the connections to the network. Notice that up to and including $1 \mathrm{~Hz}$ the phases are below about $1 \mathrm{mr}$ and the distribution apparently reflects a random type of noise with a mean near zero. However, at $4 \mathrm{~Hz}$ and above, the phases errors are clearly systemmatic and therefore likely from a different source. Above $4 \mathrm{~Hz}$ the mean phase increases with frequency. At $256 \mathrm{~Hz}$ there are errors of nearly $25 \mathrm{mr}$ in the phase data. The source of these errors is not a capacitive reactance which would yield negative phase values. Instead, the source is an inductive reactance that results in the positive phase values.

The corresponding phases for the case when the capacitors are in the network is also shown in Figure 5. Because the exact value of four electrode measurement will be a complex function of the network makeup, it is not possible to draw the same type of conclusions as was possible for the blank network where we knew that the phases were to be zero for each measurement. For this case we know that the phases should be all negative, reflecting a capacitive reactance --the current leads the voltage. Notice that at $1 \mathrm{~Hz}$ and below, the phases at each frequency are distributed around zero. Even for the parallel resistor and capacitor (case where the phase should be most negative) we can expect only $-0.6 \mathrm{mr}$ at 1 $\mathrm{Hz}$ (see calculation below) - of the same order as the noise of the instrument which appears 
to be about $\pm 0.2 \mathrm{mr}$ (from the low frequency scatter observed in this figure in the blank network). Above $1 \mathrm{~Hz}$ the measured phases clearly reflect the affect of the network reactance with most values being negative and some nearly $-150 \mathrm{mr}$. The phase at the higher frequencies appear to be some convolution of the inductive errors apparent in the blank data and the actual capacitive reactance.

The phase dependence on frequency can also be seen in Figure 6 where we plot the measured phase for just one of the four point measurements on the network and the phase calculated for a simpler two-component system. The measured value we use is the tenth reading in the measurement protocol in which the source current dipole is on nodes $1,1,3$ and $1,3,3$ and the voltage is measured on dipole 7,3,3 and 7,1,3. This measurement produced one of the larger measured phases in each data set. The simple system used for comparison is a parallel $\mathrm{R}=1000 \mathrm{ohm}$ resistor and $\mathrm{C}=1 \mathrm{micro}$ farad capacitor for which the magnitude $|Z|=R /\left(1+\omega^{2} C^{2} R^{2}\right)^{1 / 2}$ and the phase is $\theta=-\tan ^{-1} \omega C R$. Of course, the network matches this simple circuit only along the 12 sides of a single voxel so the measured and calculated values don't have to match. There is, however, a surprisingly good correspondence over the range of frequency of interest. This figure shows that the system (network and measurement device) is performing qualitatively as expected. A more quantitative evaluation will come in the next section where we examine the EIT reconstructions.

\section{Absolute Reconstructions}

Figure 7 shows the reconstruction of magnitude $|\mathrm{Z}|$ and phase $\theta$ using the $4 \mathrm{~Hz}$ data for both the blank and reactive networks. In the blank network the magnitude should be uniformly $1000 \mathrm{ohm} \mathrm{m}$ (the unit scale was set to $1 \mathrm{~m}$ ) and the phase should be uniformly $0.0 \mathrm{mr}$. In the reactive network the magnitude image should be $1000 \mathrm{ohm}$ m with a 500 ohm $m$ voxel at $5,5,3 ; 6,5,3 ; 5,6,3 ; 6,6,3 ; 5,5,4 ; 6,5,4 ; 5,6,4 ; 6,6,4$ and a $999.7 \mathrm{ohm} m$ voxel at $2,2,2 ; 3,2,2 ; 2,3,2 ; 3,3,2 ; 2,2,3 ; 3,2,3 ; 2,3,3 ; 3,3,3$. The phase image should be uniormly $0 \mathrm{mr}$ except for a $-25 \mathrm{mr}$ voxel at $2,2,2 ; 3,2,2 ; 2,3,2 ; 3,3,2 ; 2,2,3 ; 3,2,3 ; 2,3,3 ; 3,3,3$. The reconstructions are faithful to only part of this picture. The magnitude image for the blank is mostly about $1000 \mathrm{ohm} \mathrm{m}$ but a part along the $\mathrm{z}=1$ plane is as large as $3000 \mathrm{ohm} \mathrm{m}$. This same behavior is observed in the magnitude reconstruction of the reactive network. Much of the volume is about $1000 \mathrm{ohm} m$ but along the $\mathrm{z}=1$ plane the values go as high as $20,000 \mathrm{ohm} \mathrm{m}$. We believe that this anomalous behavior along $\mathrm{z}=1$ is a result of ineffective treatment of the no-current-flow boundary condition at the edge of the network in the forward finite difference calculation of CR3D.

The phase image of the blank ranges between about -3 and $0 \mathrm{mr}$. From Figure 3 we estimated an error in the raw data of about $\pm 2 \mathrm{mr}$ so that we believe this phase image is a faithful reconstruction of the data. The phase of the reactive network is mostly close to $0 \mathrm{mr}$ except for the large anomaly of about $-15 \mathrm{mr}$ centered on the reactive voxel which should be $-25 \mathrm{mr}$. We believe that this image is also a faithful reconstruction of the data although the anomaly is clearly smeared (or smoothed) over more than one voxel and the phase is only $-15 \mathrm{mr}$. Because the algorithm uses a smoothness constraint for stability we should not be surprised to see such evidence of smoothing.

\section{Comparison Reconstructions}

Often it is convenient to compare two or more EIT images in order to follow time dependent changes. We compare here the reconstructions of the blank and reactive network as though we are comparing a 'baseline' and 'after' image. First we show in Figure 8 the voxel by 
effectively remove the problem. The conductive anomaly built into the reactive network (which should image as a $-50 \%$ anomaly) is overwhelmed by the artifact which leaves about $\mathrm{a}+600 \%$ anomaly near the plane $\mathrm{z}=1$. A more careful examination, however, shows that there is an anomaly near where the conductive voxel should be located. It can be seen in the Figure 8 magnitude comparison only if voxels are illuminated in the narrow band between $-45 \%$ and $-35 \%$. Unfortunately, this result is not to useful since finding the anomaly requires knowing its location.

The results for the phase differences are more encouraging. Figure 8 shows the phase differences (not percent differences) created from the reconstructed voxels of the blank and reactive network. For this case, the expected $-25 \mathrm{mr}$ phase anomaly is clearly located and is approximately the correct volume at the $-15 \mathrm{mr}$ isocontour level.

Another method for making comparisons is the method of ratios in which individual transfer impedances are compared instead of the reconstructed voxels. To accomplish this comparison the ratio is formed from corresponding transfer impedance measurements

$$
Z_{I}^{R}=\left(Z_{I}^{\text {after }} / Z_{I}^{\text {baseline }}\right) Z_{I}^{\text {uniform }}
$$

where

$Z^{\text {after }}$ is the impedance of the Ith reading in the 'after' condition (reactive network)

$Z^{\text {baseline }}$ is the impedance of the Ith reading in the 'baseline' condition (blank network)

$Z^{\text {uniform }}$ is the impedance of the Ith reading for the case of a uniformly resistive whole space of $1 \mathrm{ohm} \mathrm{m}$. This value is calculated by numerically solving Laplace's equation on a uniform finite difference mesh of $|Z|=1 \mathrm{ohm} \mathrm{m}$ and $\theta=0 \mathrm{mr}$. When the two comparison data sets are identical then

$Z_{I}^{R}=Z_{I}^{\text {miform }}=1+j 0$

and we get a uniform image of magnitude 1 and phase $0 \mathrm{mr}$.

This type of comparison yields reconstructed images that are a quotient of magnitudes and a difference of phases. Specifically, when comparing data from the resistive and reactive networks we should get a uniform image of magnitude 1 and phase $0 \mathrm{mr}$ only where the voxels of the baseline and after cases are identical. At the resistive anomaly we have $Z^{R}$, $=0.5+\mathrm{j} 0$ and we should get a voxel of magnitude 0.5 and phase $0 \mathrm{mr}$.

At the reactive anomaly we have $Z^{R}=0.999+j(-25 \mathrm{mr})$ and we should get a voxel of magnitude 1 and phase $-25 \mathrm{mr}$.

Figure 9 shows the ratio comparison at $4 \mathrm{~Hz}$ for the resistive and reactive networks. The magnitude reconstruction (ratio of magnitudes) shows a clear anomaly of about $0.5 \mathrm{ohm} \mathrm{m}$ centered on the $500 \mathrm{ohm} m$ voxel. Notice that the major reconstruction artifact associated with the $\mathrm{z}=1$ boundary is not a problem as it was for the percent difference comparison. 
There is, however, a minor artifact along the $y=1$ plane. The ratio method for comparing data sets is effective at reducing this type of artifact but it can not eliminate them.

The ratio reconstruction produces a phase difference similarly to the phase difference shown in Figure 8 except the phases are subtracted at different stages of the processing. In the ratio images the difference between two phase measurements is reconstructed whereas in the percent difference images the difference is between two phases from reconstructed values of corresponding voxels. The ratio images in Figure 9 shows a clear anomaly at the $-15 \mathrm{mr}$ isocontour level (actual value is $-25 \mathrm{mr}$ ) centered on the capacitive reactance without any other feature in the image.

\section{Conclusions}

By examining the transfer impedance reciprocity we estimate that at $4 \mathrm{~Hz}$, Zombie magnitude and phase measurements are accurate to about $0.1 \%$ and $0.5 \mathrm{mr}$ respectively. This result is for the case where connecting cables are only about $1 \mathrm{~m}$ long and with no electrodes in the measurement circuit. We compared these estimates to reciprocity of measurements made under similar conditions except that the system was measuring on stainless steel electrodes immersed in water. In this case the impedance reciprocity at $4 \mathrm{~Hz}$ was $\mathbf{0 . 2 \%}$ for the magnitude and $1 \mathrm{mr}$ for the phase. We see that using reciprocity as a measure of accuracy the effect of electrodes is to degrade the measurement accuracy by a factor of two. We believe the difference in these two cases is a direct result from nonlinear effects of the electrochemical reaction present at the electrode-water interface during the measurement.

Accuracy of the phase is a strong function of the measurement frequency--errors increase with frequency. Between 0.0625 and $1 \mathrm{~Hz}$ the phase errors are approximately random and less than about $\pm 1 / 2 \mathrm{mr}$. However, they increase rapidly above $4 \mathrm{~Hz}$ until at $256 \mathrm{~Hz}$ the errors are as much as $30 \mathrm{mr}$ and are systematically inductive in nature. This behavior is equivalent to an inductive reactance of almost $20 \mathrm{~m} \mathrm{H}$ in series with $1000 \mathrm{ohm}$.

Reconstruction of impedance measurements into tomographs of magnitude and phase was difficult because of the boundary conditions imposed by the network. The Neuman boundary at the network edge could not be well modeled in the finite difference mesh of the forward solver, resulting in sever artifacts in the magnitude reconstructions and less serious ones the phase reconstructions.

The boundary modeling artifacts of reconstruction were moderated by comparing images of the network with and without reactive anomalies. A voxel by voxel comparison of magnitude images recovers a rather poor image of a conductive voxel-its location and magnitude are approximate and there are artifacts left in the image. Such a comparison is much better for phase images - a difference image recovers reasonably well the location and phase value of a single voxel of capacitive reactance.

We found that a better way to compare two cases is by inverting the ratio of impedance data. This method yielded a good location and magnitude for a conductive anomaly in the magnitude reconstruction although there was a small artifact left. The position and phase response of a capacitive reactance was recovered very well in the phase image.

\section{References}

Daily, W. and E. Owen, Cross-borehole resistivity tomography, Geophysics, 56, 1228$1235,1991$. 
Marshall, D. J. and T. R. Madden, 1959, Induced polarization, a study of its causes, Geophysics, 24, no. 4, pp 790-816.

Ramirez, A., W. Daily, A. Binely and D. LaBrecque, Electrical impedance tomography of known targets, J. Eng. and Environmental Geophysics, 4, 11-26, 1999.

Sumner, J. S., 1976, Principles of induced polarization for geophysical exploration, Science Publ. Co.

Sumner, J. S., 1976, Principles of induced polarization for geophysical exploration, Science Publ. Co.

\section{Figure Captions}

Figure 1. The 3D network used in this study. Notice that connection was made to the network to allow data collection to simulate a cross borehole geometry-five boreholes along each side of the network.

Figure 2. Schematic of the 3D rectilinear network. Each line represents a component-a resistor or resistor and capacitor in parallel. The coordinate system is defined by the nodes-the connection points for adjacent components. At the bottom of the figure are the individual planes with the components that connect the planes together to form the assembled rectilinear network that is depicted at the top.

Figure 3. Reciprocity of magnitude and phase for a subset of the 3D network data. (a) Percent difference between the magnitudes of two reciprocal measurements plotted against the transfer resistance magnitude. (b) Difference between the phases of two reciprocal measurements plotted against the transfer resistance phase.

Figure 4. Reciprocity of magnitude and phase for water tank data. (a) Percent difference between the magnitudes of two reciprocal measurements plotted against the transfer resistance magnitude. (b) Difference between the phases of two reciprocal measurements plotted against the transfer resistance phase.

Figure 5. Measured phases as a function of frequency from the 3D network. (a) Phases measured on the resistive network. (b) Phases measured on the reactive (capacitance) network.

Figure 6. Phase as a function of frequency for a single transfer resistance. Points are measured phase values from the $3 \mathrm{D}$ reactive network. The line is the calculated phase for a $1000 \mathrm{ohm}$ resistor and $1 \mathrm{micro}$ farad capacitor in parallel.

Figure 7. Reconstruction of impedance for the resistive network and for the reactive network. Only a few planes are shown so that behavior interior to the image block can be seen. (a) Magnitude for the uniformly resistive network of $1000 \mathrm{ohm} \mathrm{m}$ and $0 \mathrm{mr}$ and for the network with one resistive anomaly of $500 \mathrm{ohm} \mathrm{m}$. (b) Phase for the uniform resistive 
network of $0 \mathrm{mr}$ and for the reactive network of $0 \mathrm{mr}$ except with a single anomaly of -25 $\mathrm{mr}$.

Figure 8. Voxel by voxel comparisons between the $4 \mathrm{~Hz}$ reconstructions of the uniform resistive network and the nonuniform reactive network. As indicated on the color bars, some of the voxels are made transparent so that key internal features can be viewed without being obscured by the outer portions of the image block. (a-top) The magnitude images are repeated here from Figure 7. Also shown is the image of percent differences between magnitudes of corresponding voxels on a color scale spanning the range in differences. Then (far right) the color bar is adjusted to highlight the differences expected from the known component values. (b) The phase images are formed by computing the differences between phase values of corresponding voxels.

Figure 9. Data ratio comparisons between the $4 \mathrm{~Hz}$ data from the uniform resistive network and the nonuniform reactive network. As indicated on the color bars, some of the voxels are made transparent so that key features can be viewed without being obscured by the outer portions of the image block. (a) The magnitude images are formed from the magnitude of the reconstructed quotient equation 1. (b) The phase images are formed from the phase of the reconstructed quotient equation 1. 


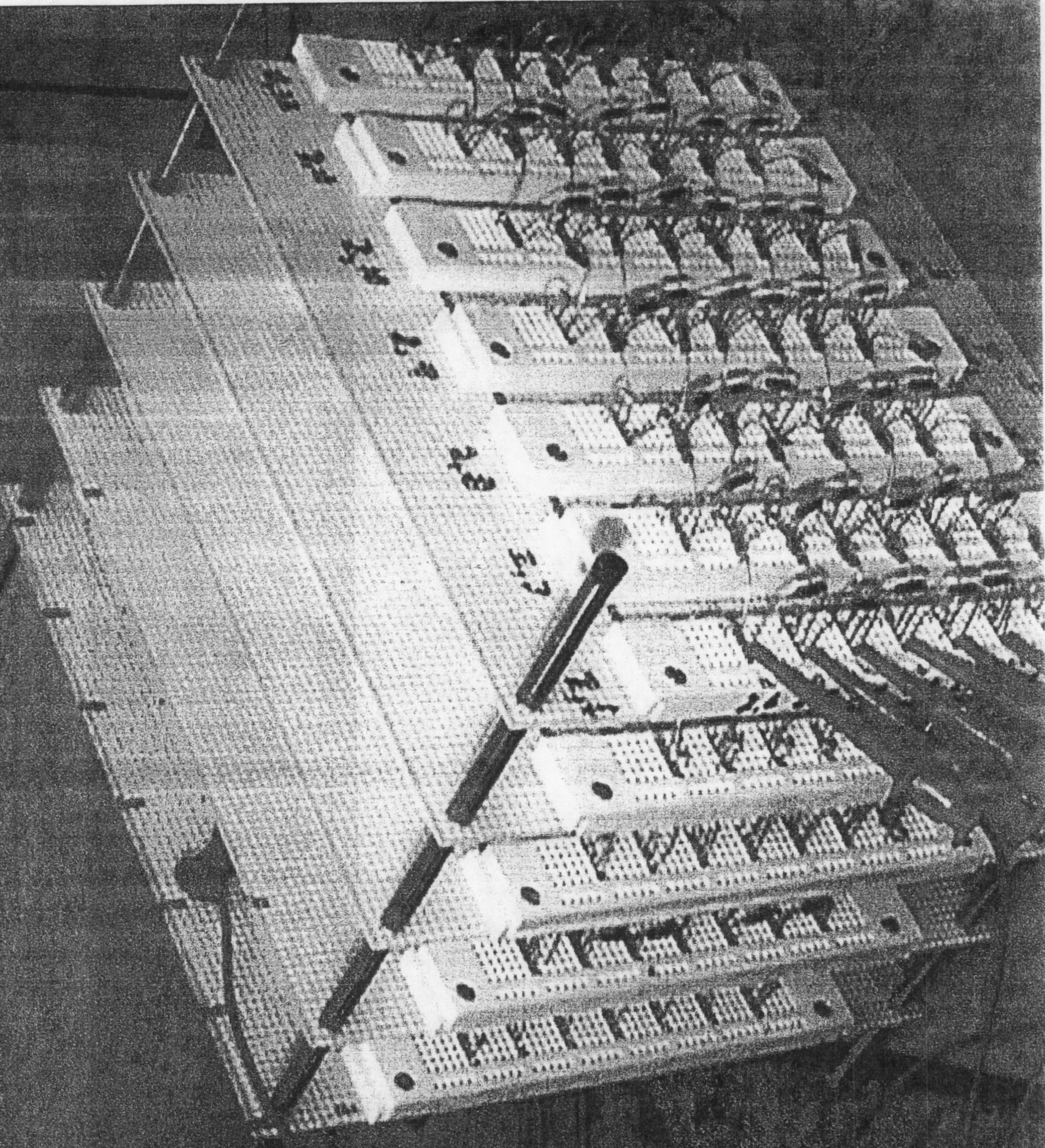





Figure 2 


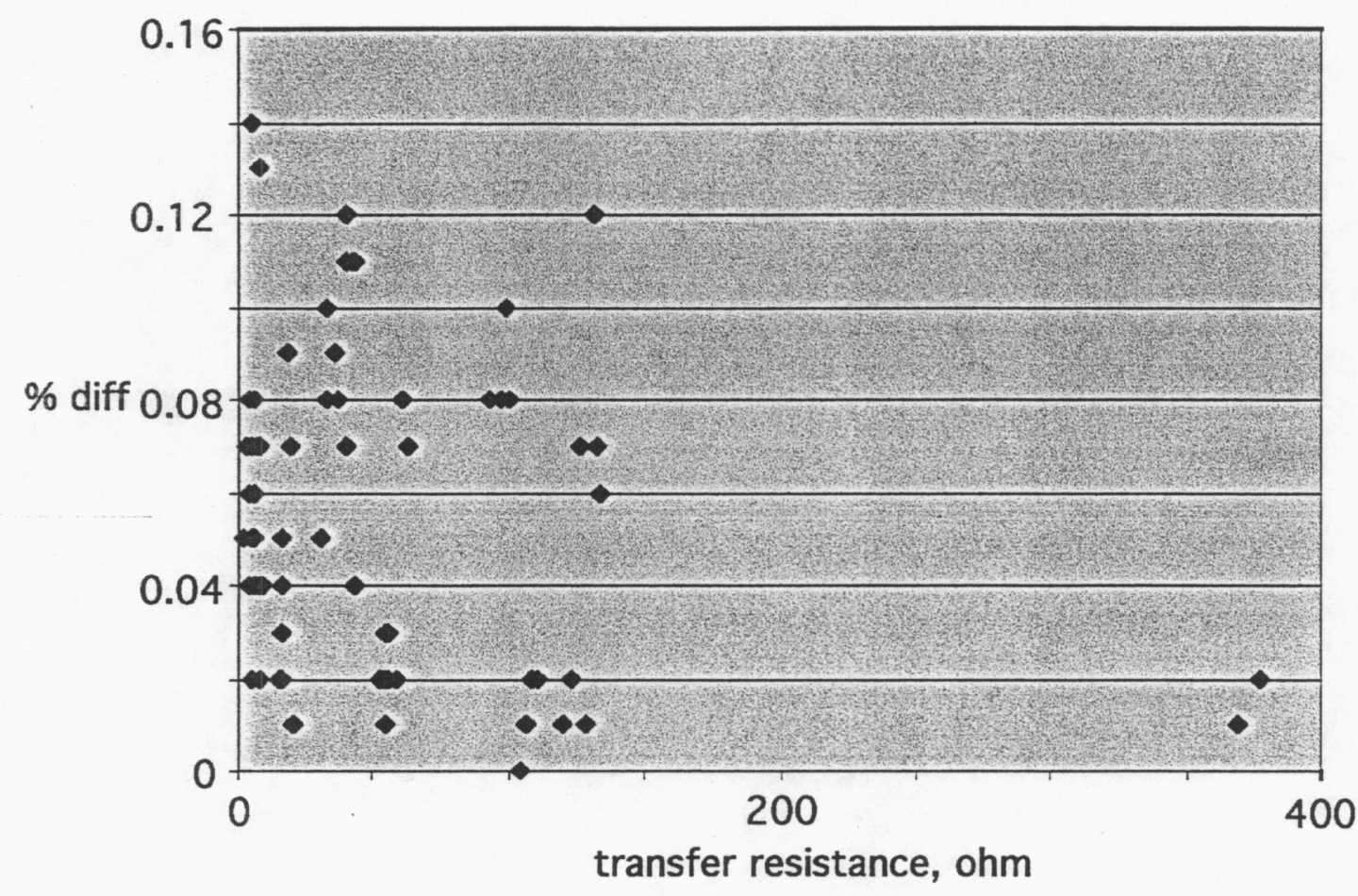

(a)

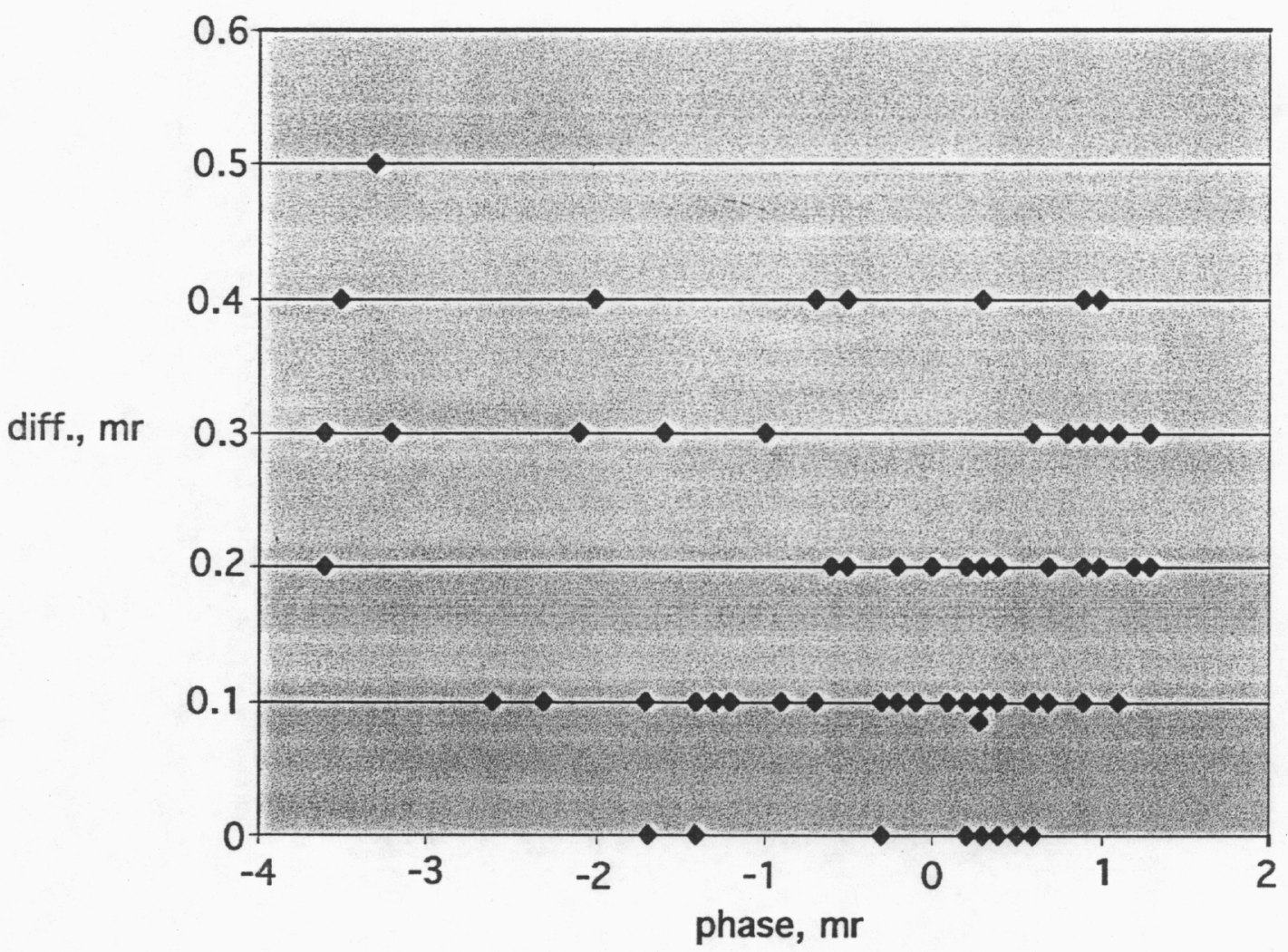

(b)

Figure 3 

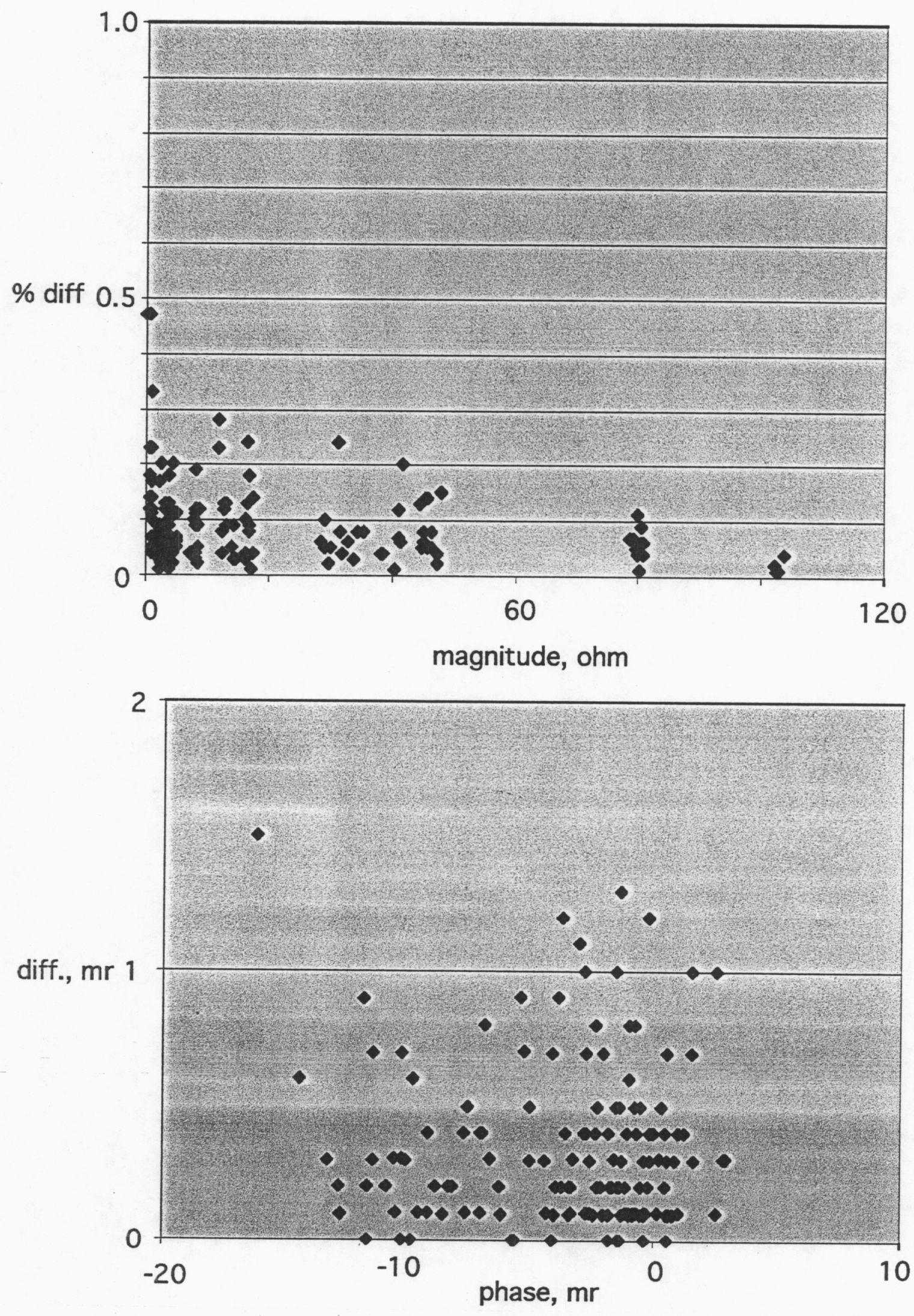

b

figure 4 

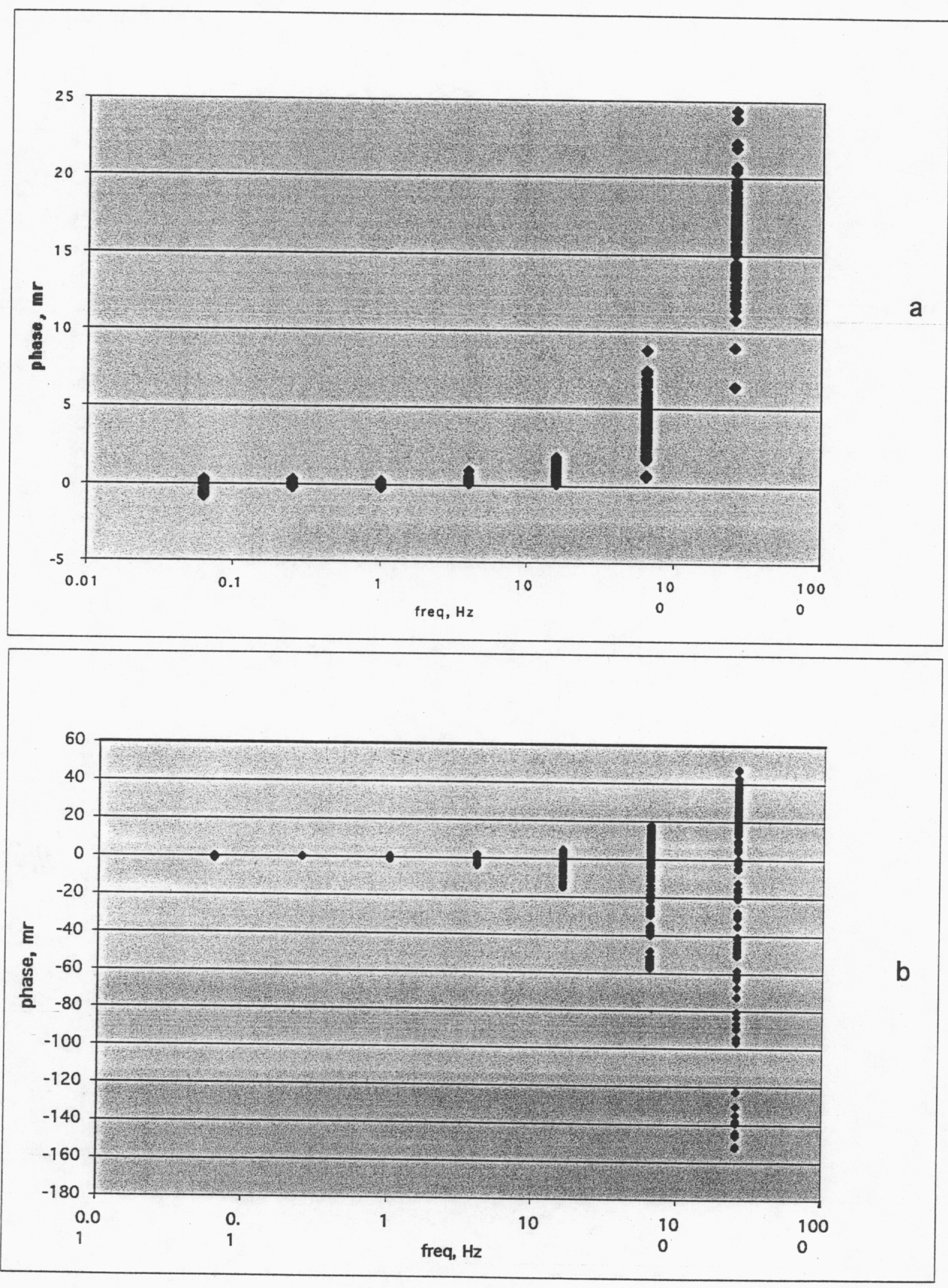

Figure 5 


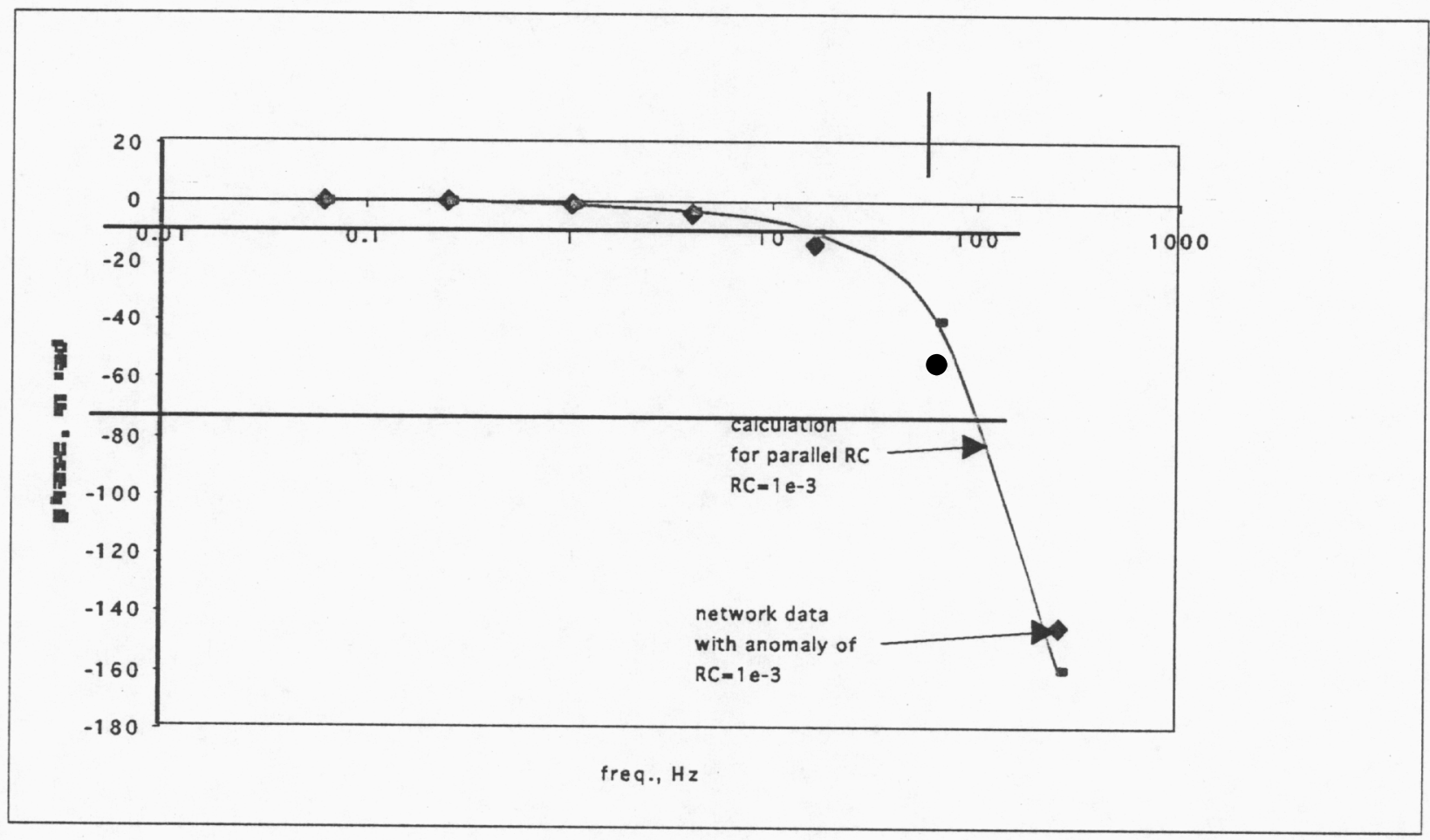

Figure 6 


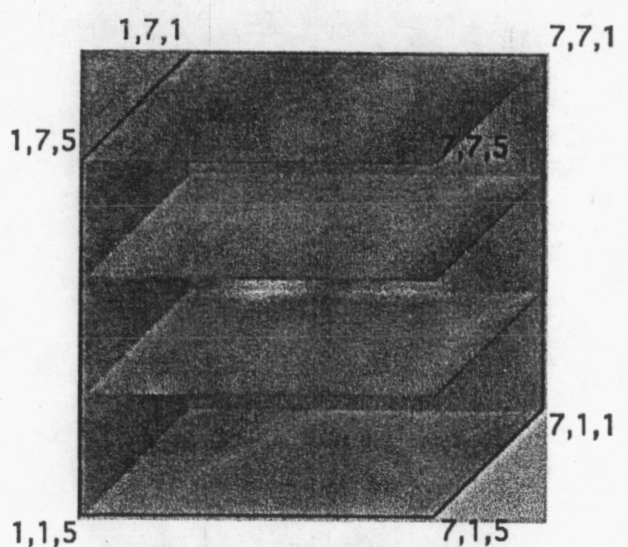

Blank, Magnitude

resistivity, ohm $\mathrm{m}$

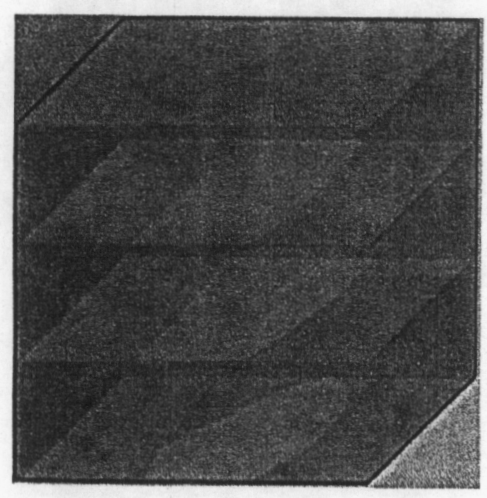

Blank, Phase

phase, blank (m rad)

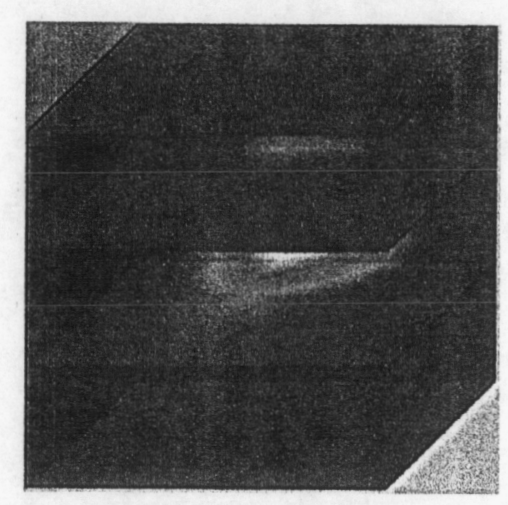

Anomaly, Magnitude

resistivity, ohm m

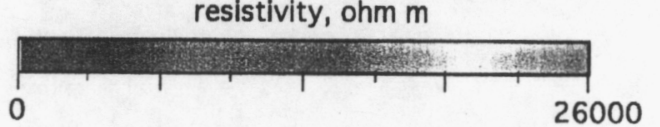




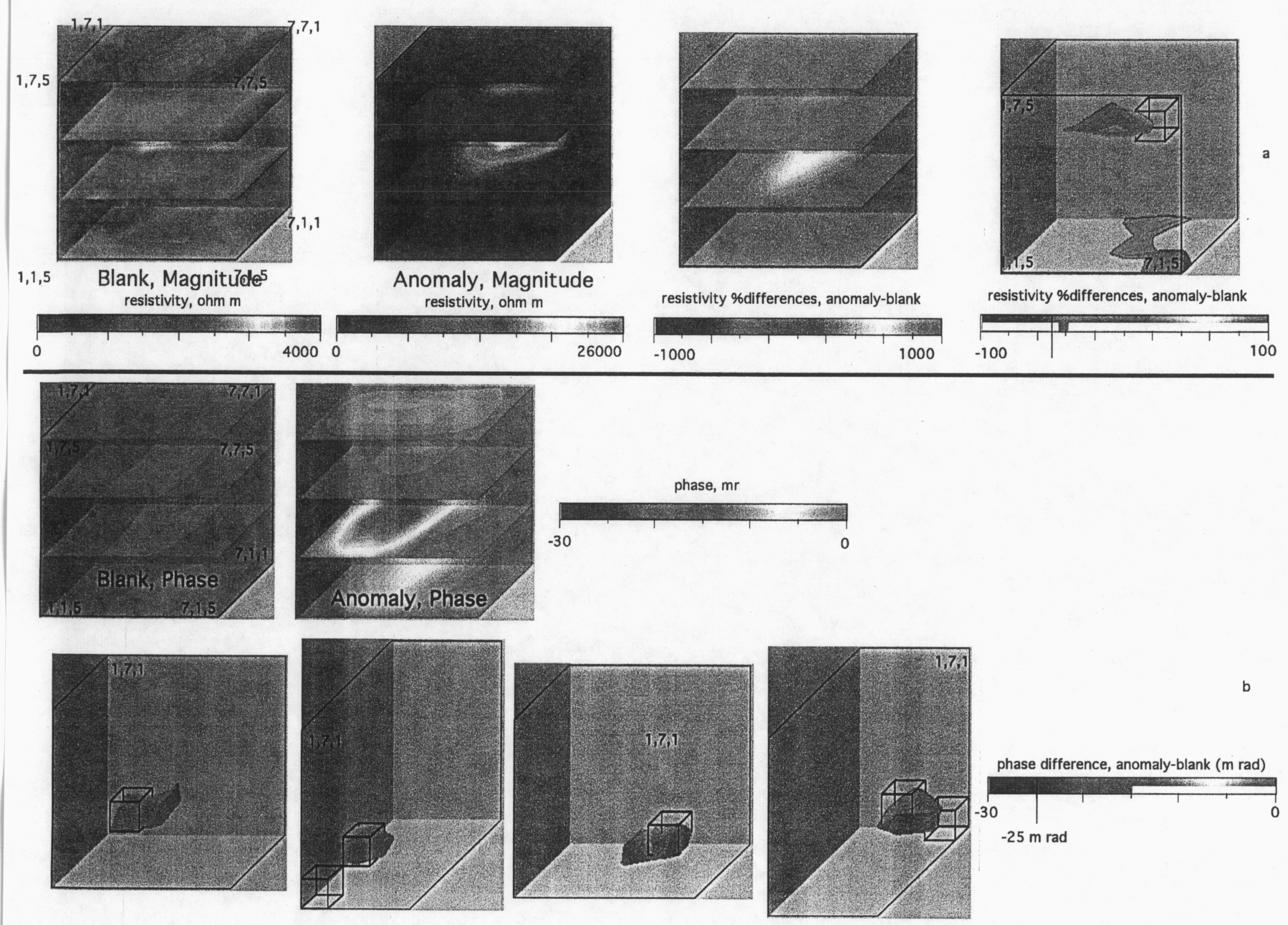

Figure 8 
